\title{
Lycophytes and ferns composition of Atlantic Forest conservation units in western Paraná with comparisons to other areas in southern Brazil
}

\author{
Mayara Lautert ${ }^{1 *}$, Lívia Godinho Temponi ${ }^{1}$, Raquel S. Viveros ${ }^{2}$ and Alexandre Salino ${ }^{2}$
}

Received: March 12, 2015 Accepted: July 6, 2015

\begin{abstract}
This study surveyed lycophyte and fern species in four forest fragments in western Paraná, Brazil, and compared them to 15 other fragments with different plant formations from the Atlantic Forest biome in southern Brazil. In total, five lycophyte species (in two families and two genera) and 98 species and two varieties of ferns (in 16 families and 38 genera) were registered in the four fragments. The most represented families were Pteridaceae ( $23 \mathrm{spp}$.), Polypodiaceae (18 spp.), Aspleniaceae (13 spp.), and Thelypteridaceae (11 spp.). Asplenium (12 spp.), Thelypteris (10 spp.), and Blechnum (seven spp.) were among the most represented genera. The occurrence of Dicksonia sellowiana was noteworthy because it was associated with seasonal semideciduous forest and is threatened in Brazil. Similarity among areas was determined by a cluster analysis (UPGMA and Sørensen's index) and the relation between similarity and geographic distance was determined through Matel's analysis. The analyses revealed greater similarity among the four study areas and, for these areas as a whole, greater similarity to fragments in Rio Grande do Sul, which is evidence that these areas have similar environmental conditions.
\end{abstract}

Keywords: Araucaria forest, pteridophytes, semideciduous forest, similarity, species richness

\section{Introduction}

Lycophytes and ferns are represented by about 13,600 species. The distribution of these groups follows a pattern known as the latitudinal gradient of diversity, where in both hemispheres, from poles to equator, the number of species per unit area increases more than 30 times (Moran 2008). However, pteridophyte richness is not regular, and around $75 \%$ of this group occurs in tropical and subtropical humid regions (Tryon \& Tryon 1982).

The Atlantic Forest is one of the 34 global "hotspots" of biodiversity (Mittermeier et al. 2004), is the second largest tropical forest in the Americas, and originally extended continuously along the Brazilian coast, to eastern Paraguay and northeastern Argentina at its southern limit (SOS Mata Atlântica 2015). In Brazil, it is regarded as one of the richest biomes in plant diversity where there are 15,782 species, of which 7,155 are endemic (Stehmann et al. 2009). Out of the total number of species in the Atlantic Forest, 840 are lycophytes and ferns (Salino \& Almeida 2009).

The high environmental diversity of the Atlantic Forest is thought to be the cause of the species richness, diver- sity, and high degree of endemism in this biome (Silva \& Casteleti 2005). Latitude varies significantly along the Atlantic Forest, which, unlike other tropical forests, stretches more than 27 degrees. Elevation is also an important factor, which ranges from sea level to $2,700 \mathrm{~m}$ (Rizzini 1992). In addition, there is longitudinal variation, where inland forests differ significantly from those near the coast; the more inland the forests are, the more seasonal they become (Oliveira-Filho \& Fontes 2000).

Species composition varies widely because of environmental diversity. MacArthur \& Wilson (1967) pointed out that geographical proximity influences floristic similarity and, according to Oliveira-Filho \& Fontes (2000), different forest formations in the Atlantic Forest have different plant species compositions. Thus, for biodiversity conservation purposes, the Atlantic Forest cannot be addressed as a homogeneous unit and there is a need to take into account the various forest types found in this biome. As defined by Veloso et al. (1991), this biome has the following plant formations: rainforest, Araucaria forest, semideciduous forest and deciduous forest, as well as associated ecosystems, such as mangroves, restinga, high

\footnotetext{
${ }^{1}$ Centro de Ciências Biológicas e da Saúde, Universidade Estadual do Oeste do Paraná, Av. Universitária, 2069, 85819-110, Cascavel, PR, Brazil ${ }^{2}$ Departamento de Botânica, Instituto de Ciências Biológicas, Universidade Federal de Minas Gerais. Antônio Carlos 6627, Pampulha, 31270-901, Belo Horizonte, MG, Brazil

* Corresponding author: lautert.m@gmail.com
} 
altitude grasslands, inland swamps, and forest enclaves in the Northeast Region.

In Paraná, the remaining Atlantic Forest is restricted to a few publicly and privately protected areas, such as Parque Nacional do Iguaçu, Parque Nacional de Ilha Grande, Parque Estadual São Camilo, Parque Estadual da Cabeça do Cachorro, Parque Estadual do Rio Guarani, and the private nature reserve Fazenda Santa Maria. Most of these areas are included in the Iguaçu-Paraná Biodiversity Corridor, located in the Iguaçu and Paraná III river basins (Tossulino et al. 2007).

The aim of this study was to survey the ferns and lycophytes in Parque Estadual Cabeça do Cachorro (PECC), Parque Estadual do Rio Guarani (PERG), Parque Nacional do Iguaçu (PNI) and Fazenda Santa Maria (FSM), and to analyze the floristic similarity among these four areas and other areas of Atlantic Forest in southeastern and southern Brazil. This study considers the hypothesis that areas with similar forest formations have a similar floristic composition, regardless of their geographical proximity (MacArthur \& Wilson 1967; Oliveira Filho \& Fontes 2000).

\section{Material and methods}

\section{Study areas}

The study was conducted in four protected areas (Fig. 1). In these regions the climate is characterized as mesothermal humid subtropical, which is CFA in Köppen's classification (Köppen 1931), with an average annual temperature of $21^{\circ} \mathrm{C}$.
Summers have an average temperature of over $22^{\circ} \mathrm{C}$ and winters have an average below $18^{\circ} \mathrm{C}$. Rainfall is well distributed throughout the year, decreasing slightly in the winter, and annual rainfall is around $1,800 \mathrm{~mm}$ (IAPAR 2015). Soils are predominantly dark red, purple, and dark brown latosols, with a deep profile and high fertility (Larach et al. 1984).

Parque Estadual Cabeça do Cachorro (PECC), located at $24^{\circ} 54^{\prime} 47^{\prime \prime} \mathrm{S} / 53^{\circ} 54^{\prime} 35^{\prime \prime} \mathrm{W}$, is $60.98 \mathrm{ha}$ and $371 \mathrm{~m}$ above sea level (IAP 2006). Fazenda Santa Maria (FSM), located at $25^{\circ} 29^{\prime} 47^{\prime \prime} \mathrm{S} / 54^{\circ} 21^{\prime} 47^{\prime \prime} \mathrm{W}$, is 250 ha and $292 \mathrm{~m}$ above sea level. Parque Nacional do Iguaçu (PNI), located between $25^{\circ} 05^{\prime} \mathrm{S} / 25^{\circ} 41^{\prime} \mathrm{W}$ and $53^{\circ} 40^{\prime} \mathrm{S} / 54^{\circ} 38^{\prime} \mathrm{W}$, is the largest fragment with an area of 185,262.5 ha and has an elevation ranging from 100 to $600 \mathrm{~m}$ (IBAMA 1999). Parque Estadual do Rio Guarani (PERG), located between $25^{\circ} 05^{\prime} \mathrm{S} / 25^{\circ} 41^{\prime} \mathrm{W}$ and $53^{\circ} 40^{\prime} \mathrm{S} / 54^{\circ} 38^{\prime} \mathrm{W}$, is 2,235 ha and 300 to $400 \mathrm{~m}$ above sea level (IAP 2002).

The dominant vegetation in the four areas is semideciduous forest; however, in Parque Nacional do Iguaçu there is also a portion that is predominantly transitional vegetation between semideciduous forest and Araucaria forest (Roderjan et al. 2002; IBGE 2012).

\section{Floristic survey}

The floristic survey was conducted between August 2010 and September 2013. In Parque Estadual Cabeça do Cachorro and RPPN Fazenda Santa Maria, the existing trails were surveyed, totaling about $3.5 \mathrm{Km}$ in each area. For the Parque Nacional do Iguaçu, 20 trails were surveyed, totaling about $50 \mathrm{~km}$. In Parque Estadual do Rio Guarani, seven

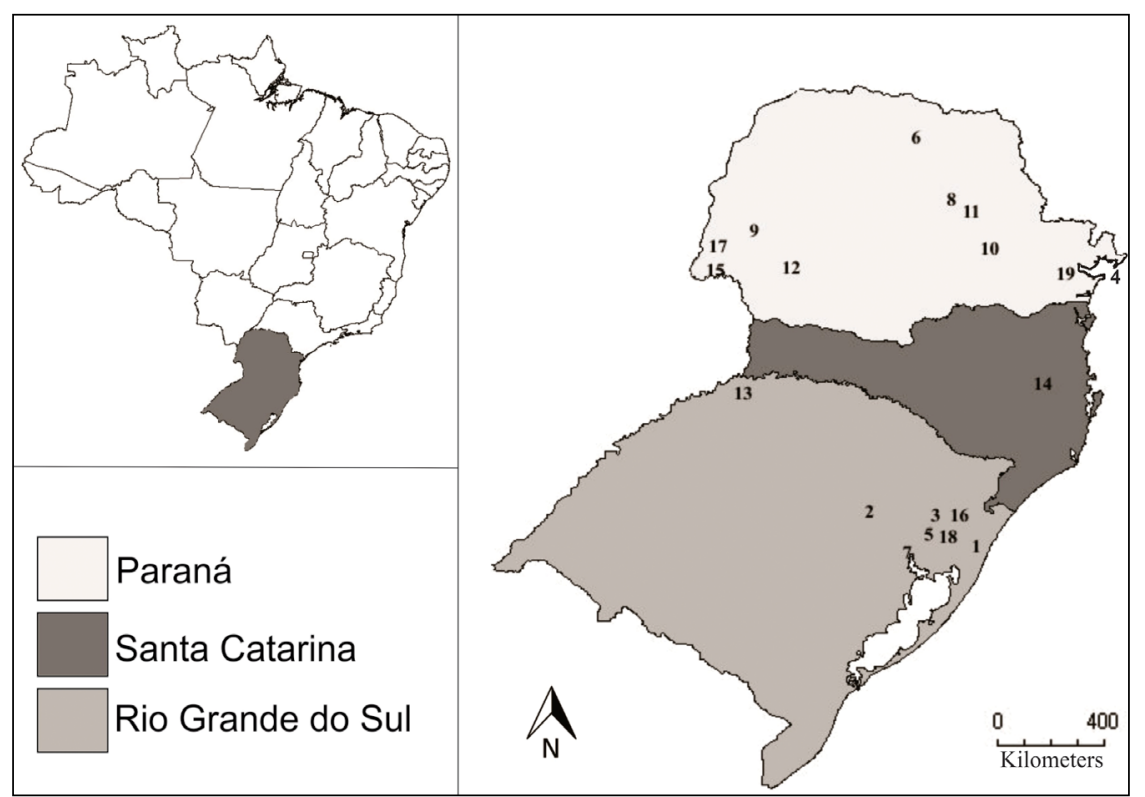

Figure 1. Locations of study areas and areas selected for the similarity analysis. 1. MB: Área de Proteção Ambiental Morro da Borússia; 2. RVT: Remnants from Vale do Taquari; 3. FLONAC: Floresta Nacional de Canela; 4. IM: Ilha do Mel; 5. LPV: Location Picada Verão; 6. MG: Mata do Godoy; 7. ME: Morro da Extrema; 8. PEcK: Parque Ecológico Klabin; 9. PECC: Parque Estadual Cabeça do Cachorro; 10. PEVV: Parque Estadual Vila Velha; 11. PEG: Parque Estadual Guartelá; 12. PERG: Parque Estadual Rio Guarani; 13. PET: Parque Estadual Turvo; 14. PNSI: Parque Nacional Serra de Itajaí; 15. PNI: Parque Nacional Iguaçu; 16. MR: Parque Natural Municipal da Ronda; 17. FSM: Fazenda Santa Maria; 18. SFP: Mata de São Francisco de Paula; 19. SMPr: Serra do Mar Paranaense. 
trails were surveyed, totaling about $17 \mathrm{~km}$. Sampling was done in an unsystematic way in order to go through all of the vegetation types. Information regarding the habitat and habit of collected plants was recorded.

The plants were collected and processed according to standard techniques for lycophytes and ferns (Bridson \& Forman 1998) and the specimens were archived at UNOP (Universidade do Oeste do Paraná) and BHCB (Thiers 2015). Additional specimens from BHCB and MBM (Thiers 2015) were included in the study.

Species were identified using the literature and herbarium specimens. For lycophytes, the classification system proposed by Kramer \& Green (1990), with modifications by Øllgaard (2012), was used. The classification of ferns was based on Smith et al. (2006), with modifications by Rothfels et al. (2012) for Woodsiaceae. Author names of taxa were abbreviated according to the International Plant Names Index (IPNI) (Brummit \& Powell 1992).

\section{Similarity}

The list of lycophyte and fern species in the sampled areas (Parque Estadual Cabeça do Cachorro, Fazenda Santa Maria, Parque Nacional do Iguaçu, and Parque Estadual do Rio Guarani) was compared to 15 other, previously published lists (Tab. 1) for Atlantic Forest areas in southern Brazil (Fig. 1).
To compare the floristic similarity, we constructed a presence/absence matrix of species (Tabs. 1-2). A cluster analysis was performed with this matrix, which resulted in a dendrogram, by using the algorithm unweighted pair-group method using arithmetic averages (UPGMA) and Sørensen's index (Bray-Curtis) (Gotelli \& Ellison 2011). To assess the deformation degree caused by the dendrogram construction, the cophenetic correlation coefficient was calculated (Hammer \& Harper 2006). Analyses were conducted using the software Palaeontological Statistics (PAST) (Hammer et al. 2001).

To test the hypothesis that closer areas are more similar regarding their composition, Mantel's test was chosen because it is a test where the predictor variable is space, measured as geographical distance (Hammer \& Harper 2006).

\section{Results and Discussion}

\section{Floristic survey}

In the four protected areas surveyed, 105 taxa (103 species and two varieties) were recorded. Of these, five species are lycophytes and 98 species and two varieties are ferns. The lycophytes are represented by two families, Selaginellaceae and Lycopodiaceae, each with one genus (Tab. 2). The ferns are distributed among 16 families and 38 genera (Tab. 2).

Table 1. List of the 19 locations included in the floristic similarity analysis of lycophytes and ferns. Location: APA: Environmental Protection Area; PR: Paraná; RPPN: Private nature reserve; RS: Rio Grande do Sul; SC: Santa Catarina. Vegetation: NG: natural grasslands; GF: General Fields; CF: Campestral Formations; DF: Deciduous Forest; SF: Semideciduous Forest; AF: Araucaria forest; GFo: Gallery Forest; RA: Rainforest; RES: Restinga; Sub: Submontane.

\begin{tabular}{|c|c|c|c|c|c|c|c|}
\hline Code & Location & State & Area (ha) & Elevation $(\mathrm{m})$ & Vegetation & $\mathrm{N}$ of species & Reference \\
\hline 1 & APA Morro da Borussia (MB) & RS & 7 & $50-398$ & $\mathrm{RA} / \mathrm{Sub}$ & 53 & Santos \& Windisch 2008 \\
\hline 2 & Remnants from "Vale do Taquari" (RVT) & RS & 90 and 160 & $500-600$ & $\mathrm{DF}$ & 56 & Lehn et al. 2009 \\
\hline 3 & Floresta Nacional de Canela (FLONAC ) & RS & 517.7 & 770 & $\mathrm{AF}$ & 58 & Schmitt et al. 2006 \\
\hline 4 & Ilha do Mel (IM) & PR & 3 & $0-148$ & $\mathrm{CF} / \mathrm{RES} / \mathrm{RA}$ & 114 & Salino et al. 2005 \\
\hline 5 & Location Picada Verão (LPV) & RS & - & $220-250$ & SF/Sub & 77 & Junior \& Rörig 2001 \\
\hline 6 & Mata do Godoy (MG) & PR & 690 & 690 & SF & 40 & Rosseto \& Vieira 2013 \\
\hline 7 & Morro da Extrema (ME) & RS & 1 & 255 & RA & 45 & Senna \& Kazmirczak 1997 \\
\hline 8 & Parque Ecológico Klabin (PEcK) & PR & 11,196 & 885 & $\mathrm{SF} / \mathrm{AF} / \mathrm{NG}$ & 121 & Sakagami 2006 \\
\hline 9 & Parque Estadual Cabeça do Cachorro (PECC) & PR & 60.98 & 370 & SF & 38 & Present study \\
\hline 10 & Parque Estadual Vila Velha (PEVV) & PR & $3,803.28$ & $800-1,100$ & $\mathrm{AF} / \mathrm{NG}$ & 151 & Schwartsburd \& Labiak 2007 \\
\hline 11 & Parque Estadual do Guartelá (PEG) & PR & 798.97 & $770-1,100$ & GF/GFo & 164 & Michelon \& Labiak 2013 \\
\hline 12 & Parque Estadual do Rio Guarani (PERG) & PR & 2 & $300-400$ & SF & 61 & Present study \\
\hline 13 & Parque Estadual do Turvo (PET) & RS & 18 & $100-400$ & SF & 66 & Brack et al. 1985 \\
\hline 14 & Parque Nacional Serra de Itajaí (PNSI) & SC & 57 & $150-940$ & RA & 185 & Gasper \& Sevegnani 2011 \\
\hline 15 & Parque Nacional do Iguaçu (PNI) & PR & $185,262.5$ & $200-600$ & $\mathrm{SSF} / \mathrm{AF}$ & 101 & Present study \\
\hline 16 & Parque Natural Municipal da Ronda (MR) & RS & 1 & 870 & $\mathrm{AF}$ & 42 & Blume et al. 2010 \\
\hline 17 & RPPN Fazenda Santa Maria (FSM) & PR & 250 & 292 & SSF & 40 & Present study \\
\hline 18 & São Francisco de Paula (SFP) & RS & 400 & 941 & $\mathrm{AF}$ & 41 & Senna \& Waechter 1997 \\
\hline 19 & Serra do Mar Paranaense (SMPr) & PR & 1,800 & $0-1889$ & RA/RES & 166 & Paciencia 2008 \\
\hline
\end{tabular}


Table 2. List of lycophyte and ferns species that occur in four protect areas in western Paraná. Voucher: ML: Mayara Lautert; PHL: Paulo Henrique Labiak; RSV: Raquel Stauffer Viveros. Habit: Ep: Epiphyte; Ru: Rupicolous; Sc: scandent; Tr: Terrestrial. Area sampled: 1. Parque Estadual Cabeça do Cachorro; 2. Fazenda Santa Maria; 3. Parque Nacional do Iguaçu; 4. Parque Estadual do Rio Guarani. • Species collected exclusively in semideciduous forest; ${ }^{*}$ Species collected exclusively in Arauracia forest.

\begin{tabular}{|c|c|c|c|c|c|c|}
\hline \multirow{2}{*}{ Taxon } & \multirow{2}{*}{ Habit } & \multirow{2}{*}{ Voucher } & \multicolumn{4}{|c|}{ Area sampled } \\
\hline & & & 1 & 2 & 3 & 4 \\
\hline \multicolumn{7}{|l|}{ ANEMIACEAE } \\
\hline Anemia raddiana Link • & $\operatorname{Tr} / \mathrm{Ru}$ & ML 326 & & & $\mathrm{X}$ & \\
\hline Anemia phyllitidis (L.) Sw. & $\operatorname{Tr}$ & ML 72 & $\mathrm{X}$ & $\mathrm{X}$ & $\mathrm{X}$ & $\mathrm{X}$ \\
\hline \multicolumn{7}{|l|}{ ASPLENIACEAE } \\
\hline Asplenium abscissum Willd. • & $\operatorname{Tr} / \mathrm{Ep}$ & ML 267 & & & $\mathrm{X}$ & $\mathrm{X}$ \\
\hline Asplenium auriculatum Sw. $\bullet$ & Ep & PHL 3760 & & & $\mathrm{X}$ & \\
\hline Asplenium brasiliense Sw. $\bullet$ & $\operatorname{Tr} / \mathrm{Ep}$ & ML 312 & $\mathrm{X}$ & $\mathrm{X}$ & $\mathrm{X}$ & $\mathrm{X}$ \\
\hline Asplenium claussenii Hieron. • & $\operatorname{Tr}$ & ML 69 & $\mathrm{X}$ & $\mathrm{X}$ & $\mathrm{X}$ & $\mathrm{X}$ \\
\hline Asplenium gastonis Fée & Ep & ML 231 & $\mathrm{X}$ & $\mathrm{X}$ & $\mathrm{X}$ & $\mathrm{X}$ \\
\hline Asplenium inaequilaterale Willd. & $\operatorname{Tr} / \mathrm{Ru}$ & ML 298 & & $\mathrm{X}$ & $\mathrm{X}$ & $\mathrm{X}$ \\
\hline Asplenium kunzeanum Klotzsch ex Rosenst. • & $\operatorname{Tr}$ & ML 370 & & & $\mathrm{X}$ & \\
\hline Asplenium mucronatum C.Presl • & Ep & ML 302 & & $\mathrm{X}$ & & \\
\hline Asplenium pulchellum Raddi • & $\operatorname{Tr}$ & ML 318 & & & $\mathrm{X}$ & $\mathrm{X}$ \\
\hline Asplenium scandicinum Kaulf. & Ep & ML 144 & $\mathrm{X}$ & $\mathrm{X}$ & $\mathrm{X}$ & $\mathrm{X}$ \\
\hline Asplenium serratum L. • & $\operatorname{Tr} / \mathrm{Ep}$ & ML 33 & $\mathrm{X}$ & $\mathrm{X}$ & $\mathrm{X}$ & $\mathrm{X}$ \\
\hline Asplenium stuebelianum Hieron. • & $\operatorname{Tr} / \mathrm{Ep}$ & RSV 137 & & & $\mathrm{X}$ & $\mathrm{X}$ \\
\hline Hymenasplenium triquetrum (N. Murak. \& R. C. Moran) L. Regalado \& Prada • & $\mathrm{Ru}$ & ML374 & & & $\mathrm{X}$ & \\
\hline \multicolumn{7}{|l|}{ ATHYRIACEAE } \\
\hline Deparia pertersenii (Kunze) M.Kato* & $\operatorname{Tr}$ & ML 286 & & & $\mathrm{X}$ & \\
\hline Diplazium ambiguum Raddi & $\operatorname{Tr}$ & ML 266 & & & $\mathrm{X}$ & $\mathrm{X}$ \\
\hline Diplazium cristatum (Desr. in Lam.) Alston & $\operatorname{Tr}$ & ML 130 & $\mathrm{X}$ & $\mathrm{X}$ & $\mathrm{X}$ & $\mathrm{X}$ \\
\hline Diplazium herbaceum Fée $\bullet$ & $\operatorname{Tr}$ & ML 306 & & & $\mathrm{X}$ & $\mathrm{X}$ \\
\hline Diplazium lindbergii (Mett.) Christ & $\operatorname{Tr}$ & ML 288 & & & $\mathrm{X}$ & $\mathrm{X}$ \\
\hline \multicolumn{7}{|l|}{ BLECHNACEAE } \\
\hline Blechnum acutum (Desv.) Mett. & $\operatorname{Tr} / \mathrm{Ep}$ & ML 118 & $\mathrm{X}$ & $\mathrm{X}$ & $\mathrm{X}$ & $\mathrm{X}$ \\
\hline Blechnum austrobrasilianum de la Sota & $\operatorname{Tr}$ & ML 264 & & & $\mathrm{X}$ & $\mathrm{X}$ \\
\hline Blechnum brasiliense Desv. $\bullet$ & $\operatorname{Tr}$ & ML 307 & $\mathrm{X}$ & $\mathrm{X}$ & $\mathrm{X}$ & $\mathrm{X}$ \\
\hline Blechnum gracile Kaulf. & $\operatorname{Tr}$ & ML 156 & & $\mathrm{X}$ & $\mathrm{X}$ & $\mathrm{X}$ \\
\hline Blechnum lanceola Sw. & $\operatorname{Tr}$ & ML 163 & & & $\mathrm{X}$ & $\mathrm{X}$ \\
\hline Blechnum occidentale L. • & $\operatorname{Tr}$ & ML 373 & & $\mathrm{X}$ & $\mathrm{X}$ & $\mathrm{X}$ \\
\hline Blechnum polypodioides Raddi & $\operatorname{Tr}$ & PHL 3737 & & & $\mathrm{X}$ & \\
\hline \multicolumn{7}{|l|}{ CYATHEACEAE } \\
\hline Alsophila setosa Kaulf. & $\operatorname{Tr}$ & ML 31 & $\mathrm{X}$ & $\mathrm{X}$ & $\mathrm{X}$ & $\mathrm{X}$ \\
\hline Cyathea atrovirens (Langsd. \& Fisch.) Domin • & $\operatorname{Tr}$ & RSV 172 & & & $\mathrm{X}$ & \\
\hline Cyathea phalerata Mart. & $\operatorname{Tr}$ & ML 280 & & & $\mathrm{X}$ & $\mathrm{X}$ \\
\hline \multicolumn{7}{|l|}{ DENNSTAEDTIACEAE } \\
\hline Dennstaedtia cicutaria (Sw.) T. Moore $\bullet$ & $\operatorname{Tr}$ & ML 101 & & & & $\mathrm{X}$ \\
\hline Dennstaedtia dissecta T. Moore $\bullet$ & $\operatorname{Tr}$ & ML 172 & & & $\mathrm{X}$ & $\mathrm{X}$ \\
\hline Dennstaedtia globulifera (Poir.) Hieron. & $\operatorname{Tr}$ & ML 315 & $\mathrm{X}$ & $\mathrm{X}$ & $\mathrm{X}$ & $\mathrm{X}$ \\
\hline Hypolepis stolonifera Fée * & $\operatorname{Tr}$ & ML 292 & & & $\mathrm{X}$ & \\
\hline \multicolumn{7}{|l|}{ DICKSONIACEAE } \\
\hline Dicksonia sellowiana Hook. & $\operatorname{Tr}$ & ML 289 & & & $\mathrm{X}$ & $\mathrm{X}$ \\
\hline
\end{tabular}


Table 2. Continuation.

\begin{tabular}{|c|c|c|c|c|c|c|}
\hline \multirow{2}{*}{ Taxon } & \multirow{2}{*}{ Habit } & \multirow{2}{*}{ Voucher } & \multicolumn{4}{|c|}{ Area sampled } \\
\hline & & & 1 & 2 & 3 & 4 \\
\hline \multicolumn{7}{|l|}{ DRYOPTERIDACEAE } \\
\hline Ctenitis submarginalis (Langsd. \& Fisch.) Ching & $\operatorname{Tr}$ & ML 147 & $\mathrm{x}$ & $\mathrm{X}$ & $\mathrm{x}$ & $\mathrm{X}$ \\
\hline Didymochlaena truncatula (Sw.) J. Sm. & $\operatorname{Tr}$ & ML 68 & $\mathrm{x}$ & $\mathrm{x}$ & $\mathrm{X}$ & $\mathrm{X}$ \\
\hline Lastreopsis effusa (Sw.) Tindale & $\operatorname{Tr}$ & ML 81 & $\mathrm{x}$ & $\mathrm{x}$ & $\mathrm{x}$ & $\mathrm{X}$ \\
\hline Megalastrum connexum (Kaulf.) A. R. Sm. \& R. C. Moran & $\operatorname{Tr}$ & ML 316 & $\mathrm{x}$ & $\mathrm{X}$ & $\mathrm{X}$ & $\mathrm{X}$ \\
\hline Megalastrum umbrinum (C.Chr.) A.R. Sm. \& R. C. Moran & $\operatorname{Tr}$ & ML 263 & & & $\mathrm{X}$ & \\
\hline Polystichum platylepis Fée * & $\operatorname{Tr}$ & ML 290 & & & $\mathrm{X}$ & \\
\hline \multicolumn{7}{|l|}{ HYMENOPHYLLACEAE } \\
\hline Didymoglossum krausii (Hook. \& Grev.) C.Presl & $\mathrm{Ru}$ & ML 252 & & & $\mathrm{X}$ & $\mathrm{X}$ \\
\hline Polyphlebium angustatum (Carmich.) Ebihara \& Dubuisson & Ep & ML 238 & & $\mathrm{x}$ & $\mathrm{X}$ & $\mathrm{X}$ \\
\hline Polyphlebium diaphanum (Kunth) Ebihara \& Dubuisson & $\mathrm{Ru} / \mathrm{Ep}$ & ML 37 & & $\mathrm{X}$ & $\mathrm{X}$ & $\mathrm{X}$ \\
\hline Vandenbochia collariata (Bosch) Ebihara \& Dubuisson & $\mathrm{Ep} / \mathrm{Ru}$ & ML 372 & & & & $\mathrm{X}$ \\
\hline \multicolumn{7}{|l|}{ LOMARIOPSIDACEAE } \\
\hline Nephrolepis cordifolia (L.) C. Presl • & $\mathrm{Ep} / \mathrm{Tr}$ & RSV 124 & & & $\mathrm{X}$ & \\
\hline \multicolumn{7}{|l|}{ LYCOPODIACEAE } \\
\hline Phlegmariurus mandiocanus (Raddi) B. Øllg. & Ep & ML 141 & & & $\mathrm{X}$ & \\
\hline \multicolumn{7}{|l|}{ LYGODIACEAE } \\
\hline Lygodium volubile Sw. $\bullet$ & $\mathrm{Sc}$ & ML 186 & & & $\mathrm{X}$ & \\
\hline \multicolumn{7}{|l|}{ OSMUNDACEAE } \\
\hline Osmunda regalis $\mathrm{L}$. & $\operatorname{Tr}$ & ML 276 & & & $\mathrm{X}$ & $\mathrm{X}$ \\
\hline \multicolumn{7}{|l|}{ POLYPODIACEAE } \\
\hline Campyloneurum austrobrasilianum (Alston) de la Sota $\bullet$ & Ep & ML 103 & & & & $\mathrm{X}$ \\
\hline Campyloneurum minus Fée $\bullet$ & Ep & RSV 255 & & & $\mathrm{X}$ & \\
\hline Campyloneurum nitidum (Kaulf.) C. Presl & Ep & ML 80 & $\mathrm{x}$ & $\mathrm{X}$ & $\mathrm{X}$ & $\mathrm{X}$ \\
\hline Microgramma lindbergii (Mett.) de la Sota $\bullet$ & Ep & ML 257 & & & $\mathrm{X}$ & \\
\hline Microgramma squamulosa (Kaulf.) de la Sota & Ep & ML 53 & $\mathrm{x}$ & $\mathrm{X}$ & $\mathrm{X}$ & $\mathrm{X}$ \\
\hline Microgramma vacciniifolia (Langsd. \& Fisch.) Copel. • & Ep & RSV 224 & & & $\mathrm{X}$ & \\
\hline Niphidium crassifolium (L.) Lellinger & Ep & ML 138 & & & $\mathrm{X}$ & $\mathrm{X}$ \\
\hline Pecluma filicula (Kaulf.) M.G.Price & Ep & ML 304 & & $\mathrm{X}$ & $\mathrm{X}$ & $\mathrm{X}$ \\
\hline Pecluma pectinatiformis (Lindm.) M. G. Price & Ep & ML 42 & & & $\mathrm{X}$ & $\mathrm{X}$ \\
\hline Pecluma robusta (Fée) M.Kessler \& A. R. Sm. • & $\operatorname{Tr} / \mathrm{Ep}$ & ML 308 & & $\mathrm{X}$ & $\mathrm{X}$ & \\
\hline Pecluma sicca (Lindm.) M. G. Price & Ep & ML 135 & $\mathrm{x}$ & $\mathrm{x}$ & $\mathrm{X}$ & $\mathrm{X}$ \\
\hline Pecluma singeri (de la Sota) M. G. Price & Ep & ML 314 & $\mathrm{x}$ & $\mathrm{X}$ & $\mathrm{x}$ & $\mathrm{X}$ \\
\hline Pecluma truncorum (Lindm.) M. G. Price & Ep & ML 25 & $\mathrm{x}$ & $\mathrm{X}$ & $\mathrm{x}$ & $\mathrm{X}$ \\
\hline Pleopeltis astrolepis (Liebm.) E. Fourn. $\bullet$ & Ep & ML 328 & & & $\mathrm{x}$ & \\
\hline Pleopeltis hirsutissima (Raddi) de la Sota & Ep & ML 43 & $\mathrm{x}$ & $\mathrm{X}$ & $\mathrm{x}$ & $\mathrm{X}$ \\
\hline Pleopeltis minima (Bory) J. Prado \& R. Y. Hirai & Ep & ML 177 & $\mathrm{x}$ & $\mathrm{X}$ & $\mathrm{x}$ & $\mathrm{X}$ \\
\hline Pleopeltis pleopeltifolia (Raddi) Alston. & Ep & ML 71 & $\mathrm{x}$ & $\mathrm{X}$ & $\mathrm{x}$ & $\mathrm{X}$ \\
\hline Serpocaulon vacillans (Link) A. R. Sm.* & $\operatorname{Tr}$ & ML 152 & & & $\mathrm{x}$ & \\
\hline \multicolumn{7}{|l|}{ PTERIDACEAE } \\
\hline Adiantopsis chlorophylla (Sw.) Fée * & $\operatorname{Tr}$ & ML 282 & & & $\mathrm{x}$ & \\
\hline Adiantopsis perfasciculata Sehnem * & $\operatorname{Tr}$ & ML 341 & & & $\mathrm{x}$ & \\
\hline Adiantopsis radiata (L.) Fée & $\operatorname{Tr}$ & ML 70 & $\mathrm{X}$ & $\mathrm{x}$ & $\mathrm{X}$ & $\mathrm{X}$ \\
\hline
\end{tabular}


Table 2. Continuation

\begin{tabular}{|c|c|c|c|c|c|c|}
\hline \multirow{2}{*}{ Taxon } & \multirow{2}{*}{ Habit } & \multirow{2}{*}{ Voucher } & \multicolumn{4}{|c|}{ Area sampled } \\
\hline & & & 1 & 2 & 3 & 4 \\
\hline Adiantum incertum Lindm. • & $\operatorname{Tr}$ & ML 260 & & & $\mathrm{X}$ & \\
\hline Adiantum obliquum Willd. • & $\operatorname{Tr}$ & RSV 152 & & & $\mathrm{X}$ & \\
\hline Adiantum pseudotinctum Hieron. & $\operatorname{Tr}$ & ML 84 & $\mathrm{x}$ & $\mathrm{X}$ & $\mathrm{X}$ & $\mathrm{X}$ \\
\hline Adiantum raddianum C. Presl & $\operatorname{Tr}$ & ML 83 & $\mathrm{X}$ & $\mathrm{X}$ & $\mathrm{X}$ & $\mathrm{X}$ \\
\hline Adiantum tetraphyllum Willd. • & $\operatorname{Tr}$ & ML 334 & & & $\mathrm{X}$ & \\
\hline Doryopteris concolor (Langsd. \& Fisch.) J. Sm. & $\operatorname{Tr}$ & ML 57 & $\mathrm{x}$ & $\mathrm{X}$ & $\mathrm{X}$ & $\mathrm{X}$ \\
\hline Doryopteris majestosa Yesilyurt & $\operatorname{Tr}$ & ML 66 & $\mathrm{x}$ & $\mathrm{x}$ & $\mathrm{X}$ & $\mathrm{X}$ \\
\hline Doryopteris pentagona Pic. Serm & $\operatorname{Tr}$ & ML 65 & $\mathrm{X}$ & $\mathrm{X}$ & $\mathrm{X}$ & \\
\hline Hemionitis tomentosa (Lam.) Raddi & $\operatorname{Tr}$ & ML 325 & & & $\mathrm{X}$ & \\
\hline Pityrogramma calomelanos (L.) Link var. calomelanos* & $\operatorname{Tr}$ & ML 278 & & & $\mathrm{X}$ & \\
\hline Pityrogramma calomelanos var. aureoflava (Hook.) Weath. ex Bailey * & $\operatorname{Tr}$ & ML 279 & & & $\mathrm{X}$ & \\
\hline Pityrogramma chaerophylla (Desv.) Link • & $\operatorname{Tr} / \mathrm{Ep}$ & PHL 3799 & & & $\mathrm{X}$ & \\
\hline Pteris brasiliensis Raddi & $\operatorname{Tr}$ & RSV 177 & & & $\mathrm{X}$ & \\
\hline Pteris deflexa Link • & $\operatorname{Tr}$ & ML 82 & $\mathrm{X}$ & & $\mathrm{X}$ & \\
\hline Pteris denticulata Sw. & $\mathrm{Ru}$ & ML 67 & $\mathrm{x}$ & $\mathrm{X}$ & $\mathrm{X}$ & \\
\hline Pteris lechleri Mett. & $\operatorname{Tr}$ & ML 63 & & & $\mathrm{X}$ & $\mathrm{X}$ \\
\hline Pteris vittata $\mathrm{L} . \bullet$ & $\mathrm{Ru}$ & ML 179 & & & $\mathrm{X}$ & \\
\hline Vittaria graminifolia Kaulf. • & Ep & ML 305 & & $\mathrm{X}$ & & \\
\hline Vittaria lineata (L.) Sm.* & Ep & ML 44 & & & $\mathrm{X}$ & \\
\hline \multicolumn{7}{|l|}{ SELAGINELLACEAE } \\
\hline Selaginella marginata (Humb. \& Bonpl. ex Willd.) Spring • & $\operatorname{Tr} / \mathrm{Ru}$ & ML 363 & & & $\mathrm{X}$ & \\
\hline Selaginella microphylla (Kunth) Spring • & $\mathrm{Tr} / \mathrm{Ru}$ & ML 140 & & & $\mathrm{X}$ & $\mathrm{X}$ \\
\hline Selaginella muscosa Spring & $\operatorname{Tr}$ & ML 361 & & & $\mathrm{X}$ & $\mathrm{X}$ \\
\hline Selaginella sulcata (Desv. ex Poir.) Spring & $\operatorname{Tr}$ & ML 58 & $\mathrm{x}$ & $\mathrm{X}$ & $\mathrm{X}$ & $\mathrm{X}$ \\
\hline \multicolumn{7}{|l|}{ TECTARIACEAE } \\
\hline Tectaria incisa Cav. $\bullet$ & $\operatorname{Tr}$ & ML 358 & & & $\mathrm{X}$ & \\
\hline \multicolumn{7}{|l|}{ THELYPTERIDACEAE } \\
\hline Macrothelypteris torresiana (Gaudich.) Ching & $\operatorname{Tr}$ & ML 241 & & & $\mathrm{X}$ & $\mathrm{X}$ \\
\hline Thelypteris abbiattiae C. F. Reed • & $\operatorname{Tr}$ & ML 77 & $\mathrm{x}$ & & $\mathrm{X}$ & \\
\hline Thelypteris amambayensis (Christ) Ponce & $\operatorname{Tr}$ & RSV 217 & & & $\mathrm{X}$ & \\
\hline Thelypteris dentata (Forssk.) E. P. St. John • & $\operatorname{Tr}$ & ML 256 & $\mathrm{X}$ & & $\mathrm{X}$ & $\mathrm{X}$ \\
\hline Thelypteris hispidula (Decne.) C. F. Reed • & $\operatorname{Tr}$ & ML 258 & & & $\mathrm{X}$ & $\mathrm{X}$ \\
\hline Thelypteris interrupta (Willd.) K. Iwats.* & $\operatorname{Tr}$ & ML 281 & & & $\mathrm{X}$ & \\
\hline Thelypteris recumbens (Rosenst.) C. F. Reed & $\operatorname{Tr}$ & ML 335 & & & $\mathrm{X}$ & $\mathrm{X}$ \\
\hline Thelypteris riograndensis (Lindm.) C. F. Reed & $\operatorname{Tr}$ & ML 291 & & & $\mathrm{X}$ & $\mathrm{X}$ \\
\hline Thelypteris rivularioides (Fée) Abbiatti * & $\operatorname{Tr}$ & ML 277 & & & $\mathrm{X}$ & \\
\hline Thelypteris scabra (C.Presl) Lellinger & $\operatorname{Tr}$ & ML 22 & $\mathrm{X}$ & $\mathrm{X}$ & $\mathrm{X}$ & $\mathrm{X}$ \\
\hline Thelypteris serrata (Cav.) Alston & $\operatorname{Tr}$ & ML 182 & & & $\mathrm{X}$ & \\
\hline
\end{tabular}

Of the four areas, the fragment with the largest number of species was Parque Nacional do Iguaçu (101 species), followed by Parque Estadual do Rio Guarani ( 60 species), the private nature reserve Fazenda Santa Maria (40 species), and Parque Estadual Cabeça do Cachorro (38 species) (Tab. 2). The species richness found in the four areas is similar to that reported in studies conducted in areas with the same vegetation type, especially in southern Brazil (Schmitt et al. 2006; Lehn et al. 2009).
The most representative families were Pteridaceae (22 taxa), Polypodiaceae (17), Aspleniaceae (13), and Thelypteridaceae (11). Together, these four families account for $61 \%$ of recorded taxa. Other floristic surveys in forest formations of the Atlantic Forest had similar results (Melo \& Salino 2002; Santos \& Windisch 2008; Salino \& Almeida 2009).

Species-rich genera were Asplenium (12 species), Thelypteris (10), Blechnum (seven), and Pecluma (six). Higher diversity of these taxa corroborates results obtained by other 
floristic surveys in forest formations in the Atlantic Forest (Melo \& Salino 2002; Santos \& Windisch 2008; Salino \& Almeida 2009; Gasper et al. 2012). Together, these four genera represent $33 \%$ of the registered taxa. It was noted that 19 genera are represented by only one species and 15 are represented by a few species (from two to five) (Tab. 2).

Most of the registered species (about 60\%) are widespread and have been recorded in other areas in Paraná (Dittrich et al. 2005; Salino et al. 2005; Sakagami 2006; Schwartsburd \& Labiak 2007; Michelon \& Labiak 2013). The remaining 42 species (40\%) are restricted to particular vegetations. This is possibly due to the influence of environmental factors, such as soil type, physical structure, and microclimate, which usually affect species distributions (Zuquim et al. 2012). Of the four areas studied, PNI is the only fragment that has two forest formations, where 11 taxa $(10.47 \%)$ were registered only in Araucaria forest and 31 species $(28.57 \%)$ were collected only in semideciduous forest (Tab. 2).

Regarding geographical distribution, $76 \%$ of the taxa are well distributed in the Neotropics, $11 \%$ are Pantropical species and $13 \%$ are species restricted to Brazil: Asplenium kunzeanum, A. pulchellum, Cyathea atrovirens, C. phalerata, Diplazium herbaceum, Hypolepis stolonifera, Megalastrum connexum, Pecluma sicca, P. truncorum, Pleopeltis pleopeltifolia, Polyphlebium diaphanum, Pityrogramma chaerophylla, Serpocaulon vacillans, and Thelypteris recumbens (Tab. 2); the latter has a rather restricted distribution and is endemic to southern Brazil (Prado \& Sylvestre 2015). There was a predominance of widely distributed species. Possibly, the absence of local endemic species is due to the location of the study areas because they are further inland and south, with a subtropical climate, which are characteristics that are not typical of endemism (Given 1993; Moran 2008).

Dicksonia sellowiana is a threatened species (Santiago et al. 2013) that usually occurs in association with Araucaria forest and rainforest at elevations between 600 and 2,200 $\mathrm{m}$ (Santiago et al. 2013). However, it may occur in deciduous forest (Gasper et al. 2011) and gallery forest (Salino \& Viveros 2012). In this study this is species was observed both in Araucaria forest (PNI) and in semideciduous forest, noteworthy, its first record (PERG) between 300 and $400 \mathrm{~m}$ altitudinal height.

Five species (Macrothelypteris torresiana, Nephrolepis cordifolia, Thelypteris dentata, Pteris vittata, and Deparia petersenii) found in the study areas (two species in PERG, one in PECC, and five in PNI) are exotic or naturalized (Moro et al. 2012), of extra-American origin, and occur spontaneously or sub-spontaneously in Brazil.

Adiantopsis perfasciculata and Blechnum lanceola are new records for the state of Paraná. Adiantopsis perfasciculata was cited only for Central Brazil, the state of Minas Gerais (Figueiredo \& Salino 2005; Prado 2015) and, in the South Region, the states of Rio Grande do Sul and Santa Catarina (Sehnem 1972; Gasper et al. 2012). Blechnum lanceola was previously recorded for the Central, Southeast, and South regions of Brazil, but in the latter region it was only known from Rio Grande do Sul (Dittrich 2005; Dittrich \& Salino 2015).

Regarding growth form (Page 1979; Lellinger 2002), lycophytes and ferns are predominantly herbaceous. In this study, four species were arborescent (Alsophila, Cyathea [Cyathea atrovirens and C. phalerata], and Dicksonia) and one was subarborescent (Blechnum brasiliense). Regarding habit, 65 were classified as only terrestrial (62\%), 21 as epiphytes (20\%), four as rupicolous (3.8\%), and one as scandent $(0.95 \%)$. Eleven species $(10.47 \%)$ had more than one growth habit (Tab. 2). The growth-form percentages are in accordance with the representativeness registered in other studies, such as Melo \& Salino (2002) and Kersten (2010), for epiphytes in semideciduous forest.

\section{Similarity}

The cluster analysis (Fig. 2) showed high statistical significance and the cophenetic correlation coefficient was 0.8758 , indicating that distortion between the similarity matrix and multidimensional space shown in the dendrogram was small (Hammer \& Harper 2006).

The dendrogram shows the formation of two groups. A larger group (group I) comprises 10 areas in Paraná, including the studied areas, one area in Santa Catarina, and five areas in Rio Grande do Sul. The second group (group II) comprises two areas in Rio Grande do Sul (LPV and PET) and one in Paraná (MG) (Fig. 2).

According to Mantel's analysis, differences in floristic composition between selected areas are not related to geographical distance because the test showed that geographical distance between locations was negative and strongly insignificant $(\mathrm{p}=0.432[\mathrm{p}>0.01]$ and $\mathrm{r}=0.0579)$.

The fragment São Francisco de Paula (SFP) does not occur with any other group, behaving as an outlier in the analysis. This fragment is located in an urban area in Rio Grande do Sul and more than half of the species from this survey (about 25) are different from those found in the studied areas.

Within group I, there are two subgroups (A and B). In subgroup A, the fragments PEcK (PR), PEVV (PR), PEG (PR), SMPr (PR), PNSI (SC) cluster on one branch and IM $(\mathrm{PR})$ is alone on a separate branch.

Possible reasons why Ilha do Mel (PR) differs from the other areas are its location, elevation above sea level, due to the fact that it is an island (Tab. 1), and because it has a forest formation influenced by restinga (Dzwonko \& Kornás 1994).

Branch A1 is a cluster of the areas PEcK (PR), PEVV (PR) and PEG (PR), which are about $65 \%$ similar in floristic composition (Fig. 2). These three locations share the same vegetation type (Araucaria forest and natural grasslands) (Tab. 1) and are geographically close, which suggests that both of these aspects are strong influences for this cluster. 


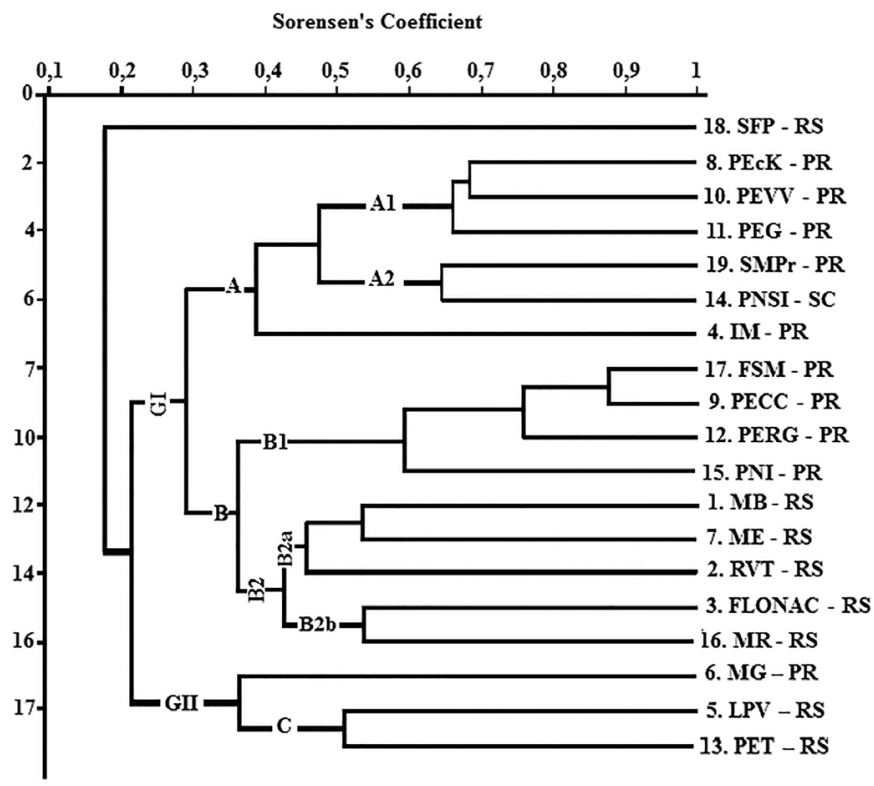

Figure 2. Similarity dendrogram of lycophyte and fern species among 19 Atlantic Forest areas in southern Brazil (using Sørensen’s Index and UPGMA algorithm). PR: Paraná; RS: Rio Grande do Sul; SC: Santa Catarina. 1. MB: Área de Proteção Ambiental Morro da Borússia; 2. RVT: Remnants from Vale do Taquari; 3. FLONAC: Floresta Nacional de Canela; 4. IM: Ilha do Mel; 5. LPV: Localidade Picada Verão; 6. MG: Mata do Godoy; 7. ME: Morro da Extrema; 8. PEcK: Parque Ecológico Klabin; 9. PECC: Parque Estadual Cabeça do Cachorro; 10. PEVV: Parque Estadual Vila Velha; 11. PEG: Parque Estadual Guartelá; 12. PERG: Parque Estadual Rio Guarani; 13. PET: Parque Estadual Turvo; 14. PNSI: Parque Nacional Serra de Itajaí; 15. PNI: Parque Nacional Iguaçu; 16. MR: Parque Natural Municipal da Ronda; 17. FSM: Fazenda Santa Maria; 18. SFP: Mata de São Francisco de Paula; 19. SMPr: Serra do Mar Paranaense.

Branch A2 consists of the fragments SMPr (PR) and PNSI (SC), which are about $62 \%$ similar and, although geographically more distant, share the same vegetation type (rainforest).

It is noteworthy that areas from subgroup A (PEcK, PEVV, PEG, SMPr, PNSI, IM) are the most representative in species number because they are located in the far eastern, coastal part of the country (mainly in Serra do Mar) that has unique features, such as the influence of humid winds coming from the ocean and heavy rainfall throughout the year (Pellegatti \& Galvani 2010). According to Page (1979), environments with a higher occurrence of lycophytes and ferns are very humid and tropical, and have no dry periods throughout the year.

Subgroup B comprises nine fragments, FSM (PR), PECC (PR), PERG (PR), PNI (PR), MB (RS), ME (RS), RVT (RS), FLONAC (RS) and MR (RS), and two branches (B1 and B2).

Branch B1 is exclusively formed by the fragments of this study, FSM, PECC, PERG and PNI (Fig. 2). The fragments FSM and PECC have the highest similarity rate, with about $90 \%$ of the same species, and these two areas are $85 \%$ similar to the species from PERG (Fig. 2). The floristic composition of $\mathrm{PNI}$ is $60 \%$ similar to the other three areas combined (Fig. 2).

As found by Mantel's test and the similarity analysis, PNI differs the most for these fragments even though it is geographically closer to FSM (Fig. 1). This might be because PNI is large and has two forest formations. Thus, also in the four areas under study in western Paraná, the greatest similarity was found between fragments with the same forest formation. Additionally, other factors could influence this similarity, such as environmental heterogeneity (Jones et al. 2006; Gasper et al. 2012) and disturbance history (Jones et al. 2008; Nóbrega et al. 2011).

Branch B2 is exclusively formed by the fragments from Rio Grande do Sul (MB, ME, RVT, FLONAC, MR). These areas are mainly Araucaria forest, except for MB, which consists of rainforest, and RVT, which is deciduous forest. Branch B2 comprises two clusters (B2a and B2b), and branch B2a comprises MB, ME and RVT. The floras of fragments $\mathrm{MB}$ and $\mathrm{ME}$ are $54 \%$ similar and these fragments, when compared to the fragment RVT, are $45 \%$ similar; RVT is the least similar. Branch B2b comprises fragments FLONAC and MR, which are $54 \%$ similar (Fig. 2). The difference of these five fragments in relation to the other areas is evidence that they are grouped according to forest formation (Araucaria forest).

In the group II there is a union of fragments that only have semideciduous forest (MG, LPV and PET). Subgroup $\mathrm{C}$ is formed by the fragments LPV and PET, which are 50\% similar, and fragment MG is further from the latter two, with about $35 \%$ similarity in its floristic composition.

The occurrence of 2 clusters with areas of semideciduous forest (GI and B1), in different branches, could be explained by how the data was collected during the studies of these areas. The studies related to the three areas of group GI were surveys of the entire vascular flora, not specifically lycophytes and ferns, which could have resulted in a sub- 
sampling of the groups. This is an important bias to consider in relation to the composition of species lists.

Based on the hypothesis proposed, when analyzing the cluster of areas in this study with areas in Rio Grande do Sul (subgroup B), a possible explanation for the existence of similarity might be the influence of Araucaria forest on the species composition within the areas of this study, especially because western Paraná is a transition area between this forest formation and semideciduous forest. There could also be a relationship between the longitude and latitude of these areas, as found for continental inland forests compared to those close to the coast, because the more inland the forests are the more seasonal they become (Oliveira-Filho \& Fontes 2000). Since humidity and temperature vary significantly relative to longitude and latitude, for the various phytophysiognomies in the Atlantic Forest, it is possible that areas located further west and further south are less influenced by humidity and have similar environmental conditions.

The results of this study support the hypothesis that areas with similar forest formations have a similar floristic composition, regardless of their geographical proximity. This is supported by evidence in subgroup A, where IM was the least similar fragment, which was mainly due to the presence of restinga in this area (Fig. 2). The hypothesis is also supported in subgroup B, where fragments FSM and PNI are geographically close (Fig. 1) but are only $60 \%$ similar in floristic composition (Fig. 2) because Araucaria forest occurs only in PNI.

The results of the similarity analysis reaffirm the fact that species composition is one of the determining factors in the plant community structure present in each forest type within a biome. Patterns found in this study were also found by Nóbrega et al. (2011), Souza et al. (2012), Salino et al. (2013), which supports the hypothesis in this work and the results in the dendrogram.

The remaining Atlantic Forest in western Paraná is known only from a few protected areas and there is a deficiency of pteridophyte studies for this region because the diversity of this group is concentrated in other areas of the state (Cislinski 1996; Dittrich et al.1999; Borgo \& Silva 2003). The species richness of the four areas, here surveyed, is lower compared with eastern regions. This was expected, since the rainforests in the east are richer than seasonal forests. However, this study contributed to the knowledge of lycophytes and ferns because little is known, based on the literature and herbarium collections, about the occurrence and distribution of species of these plants in western Paraná. Therefore, these new data are important for the conservation and management of the remaining Atlantic Forest in Brazil.

\section{Acknowledgments}

The authors thank IAP and ICMBio for permission to collect data in the study areas; MCT/CNPq/MEC/CAPES/ PROTAX 52/2010, Protocol 562240/2010-1, for the scholarship granted to the first author; and the curators of BHCB,
MBM and UPCB for their assistance and for the specimen loans. The authors thank A. Leyva and N. Smith that helped with English editing of the manuscript. L. G. Temponi thanks Fundação Araucaria and A. Salino thanks $\mathrm{CNPq}$ for the productivity grants.

\section{References}

Blume M, Fleck R, Schmitt JL. 2010. Riqueza e composição defilicíneas e licófitas em um hectare de Floresta Ombrófila Mista no Rio Grande do Sul, Brasil. Revista Brasileira de Biociências 8: 336-341.

Borgo M, Silva SM. 2003. Epífitos vasculares em fragmentos de Floresta Ombrófila Mista. Revista Brasileira de Botânica 26: 391-401.

Brack P, Bueno RM, Falkenberg DB, Paiva MRC, Sobral M, Stehmann JR. 1985. Levantamento Florístico do Parque Estadual do Turvo, Tenente Portela, Rio Grande do Sul, Brasil. Roessléria 7: 69-95.

Bridson D, Forman L. 1998. The herbarium Habdbook. London, The Royal Botanic Garden Kew.

Brummit RK, Powell CE. 1992. Author of plants names. London, The Royal Botanic Garden Kew.

Cislinski J. 1996. O gênero Diplazium Sw. (Dryopteridaceae, Pteridophyta) no Estado do Paraná, Brasil. Acta Botanica Brasilica 10: 59-77.

Dittrich VAO. 2005. Estudos Taxonômicos no gênero Blechnum L. (Pteridophyta: Blechnaceae) para as regiões Sudeste e Sul do Brasil. PhD Thesis, Universidade Estadual Paulista Júlio de Mesquita Filho, Brazil.

Dittrich VAO, Kozera C, Menezes-silva S. 1999. Levantamento florístico dos epífitos vasculares do Parque Barigüí, Curitiba, Paraná, Brasil. Iheringia Série Botânica 52: 11-21.

Dittrich VAO, Salino A. 2015. Blechnaceae in Lista de Espécies da Flora do Brasil. Jardim Botânico do Rio de Janeiro. <http://floradobrasil.jbrj. gov.br/jabot/floradobrasil/FB90813>. 05 Mar. 2015.

Dittrich VAO, Waechter JL, Salino A. 2005. Species richness of pteridophytes in a montane Atlantic rain forest plot of Southern Brazil. Acta Botanica Brasilica 19: 519-525.

Dzwonko Z, Kornás J. 1994. Patterns of species richness and distribution of pteridophytes in Rwanda (Central Africa): a numerical approach. Journal of Biogeography 21: 491-501.

Figueiredo JB, Salino A. 2005. Pteridófitas de quatro Reservas Particulares do Patrimônio Natural ao sul da região metropolitana de Belo Horizonte, Minas Gerais, Brasil. Lundiana 6: 83-94.

Gasper AL, Salino A, Vibrans AC, et al. 2012. Pteridófitas de Santa Catarina: um olhar sobre os dados do Inventário Florístico Florestal de Santa Catarina, Brasil. Acta Botanica Brasilica 26: 421-434.

Gasper AL, Sevegnani L. 2011. Lycophyta e samambaias do Parque Nacional da Serra do Itajaí, Vale do Itajaí, SC, Brasil. Hoehnea 37: 755-767.

Gasper AL, Sevegnani L, Vibrans AC, et al. 2011. Inventário de Dicksonia sellowiana Hook. em Santa Catarina. Acta Botanica Brasilica 25: 776-784.

Given DR. 1993. Changing Aspects of Endemism and Endangerment in Pteridophyta. Journal of Biogeography 20: 293-302.

Gotelli NJ, Ellison AM. 2011. Princípios de estatística em ecologia. Porto Alegre, Artmed.

Hammer Ø, Harper DAT. 2006. Paleontological data analysis. Oxford, Blackwell.

Hammer Ø, Harper DAT, Ryan DT. 2001. PAST: palaeontological statistics software package for education and data analysis. Palaeontologia Electronica 4: 1-9.

IAP - Instituto Ambiental do Paraná. 2002. Plano de Manejo do Parque Estadual do Rio Guarani. Publisher: http://www.iap.pr.gov.br/modules/conteudo/conteudo.php?conteudo=1224. Access 05 march 2015.

IAP - Instituto Ambiental do Paraná. 2006. Plano de Manejo do Parque Estadual Cabeça do Cachorro. http://www.iap.pr.gov.br/modules/ conteudo/conteudo.php?conteudo=1219. 05 Mar. 2015.

IAPAR - Instituto Agronômico do Paraná. 2015. Cartas Climáticas do Paraná. http://200.201.27.14/Sma/Cartas_Climaticas/Cartas_Climaticas. htm. 05 Mar. 2015. 
IBAMA - Instituto Brasileiro do Meio Ambiente e dos Recursos Naturais Renováveis. 1999. Plano de Manejo do Parque Nacional do Iguaçu. http://www.ibama.gov.br/siucweb/ unidades parna/planos_de_manejo/17/html/index.htm. 05 Mar. 2015.

IBGE - Instituto Brasileiro de Geográfica e Estatística. 2012. Manual técnico da vegetação brasileira. Rio de Janeiro, IBGE.

Jones MM, Tuomisto H, Clark DB, Olivas P. 2006. Effects of mesoscale environmental heterogeneity and dispersal limitation o floristic variation in rain forest ferns. Journal of Ecology 94:181-195.

Jones MM, Tuomisto H, Olivas CP. 2008. Differences in the degree of environmental control large and small tropical plants: just a sampling effect? Journal of Ecology 96: 357-377.

Junior AS, Rörig, JFS. 2001. Estudos Florísticos-Ecológicos das Pteridófitas da localidade de Picada Verão, Sapiranga - RS. Pesquisas, Botânica 51: 137-145.

Kersten RA. 2010. Epífitas vasculares - Histórico, participação taxonômica e aspectos relevantes, com ênfase na Mata Atlântica. Hoehnea 37: 9-38.

Köppen W. 1931. Grundriss der Klimakunde. Berlin, Walter de Gruyter.

Kramer KU, Green PS. 1990. The Families and Genera of Vascular Plants. Pteridophytes and Gymnosperms. Berlin, Springer-Verlag.

Larach JO, Cardoso A, Carvalho AP, Hochmuler DP, Fasolo PJ, Rauen MJ. 1984. Levantamento de Reconhecimento dos Solos do Estado do Paraná. Londrina, EMBRAPA / IAPAR.

Lehn CR, Leuchtenberger C, Hansen MAF. 2009. Pteridóftas ocorrentes em dois remanescentes de Floresta Estacional Decidual no Vale do Taquari, Estado do Rio Grande do Sul, Brasil. Iheringia Série Botânica 64: 23-31.

Lellinger D. 2002. A modern multilingual glossary for taxonomic pteridology. Pteridologia 3: 1-263

MacArthur RH, Wilson EO. 1967. The Theory of Island Biogeography. Princeton, Princeton University Press.

Melo LCN, Salino A. 2002. Pteridófitas de duas áreas de floresta da Bacia do Rio Doce no Estado de Minas Gerais, Brasil. Lundiana 3: 129-139.

Michelon C, Labiak PH. 2013. Samambaias e licófitas do Parque Estadual do Guartelá, PR, Brasil. Hoehnea 40: 191-204.

Mittermeier RA, Robles-Gil P, Hoffmann M, et al. 2004. Hotspots revisited: Earth's biologically richest and most endangered terrestrial ecoregions. Mexico City, CEMEX/ Agrupación Sierra Madre.

Moran RC. 2008. Diversity, Biogeography and floristics. In: Ranker TA, Haufler CH. (eds.) Biology and Evolution of Ferns and Lycophytes. Cambridge, Cambridge University Press. p. 367-394.

Moro MF, Souza VC, Oliveira-Filho AT, et al. 2012. Alienígenas na sala: o que fazer com espécies exóticas em trabalhos de taxonomia, florística e fitossociologia? Acta Botanica Brasilica 26: 991-999.

Nóbrega GA, Eisenlohr PV, Paciencia MLB, Prado J, Aidar MPM. 2011. A composição florística e a diversidade de pteridófitas diferem entre a Floresta de Restinga e a Floresta Ombrófila Densa das Terras Baixas do Núcleo Picinguaba/PESM, Ubatuba/SP? Biota Neotropica 11: 153-164.

Oliveira-Filho AT, Fontes MAL. 2000. Patterns of floristic differentiation among Atlantic forest in Southeastern Brazil and the influence of climate. Biotropica 32: 793-810.

Øllgaard B. 2012. Nomenclatural changes in Brazilian Lycopodiaceae. Rodriguésia 63: 479-482.

Paciencia MLB. 2008. Diversidade de Pteridófitas em gradientes de altitude na Mata Atlântica do Estado do Paraná. PhD Thesis, Universidade de São Paulo, Brazil.

Page CN. 1979. Experimental aspects of fern ecology. In: Dyer AF. (ed.) The Experimental Biology of ferns. London, Academic Press. p. 551-589.

Pellegatti CHG, Galvani E. 2010. Avaliação da precipitação na Serra do Mar - SP em eventos de diferentes intensidade e duração. GEOUSP - Espaço e Tempo 27: 147-158.

Prado J, Sylvestre L. 2015. Samambaias e Licófitas in Lista de Espécies da Flora do Brasil. Jardim Botânico do Rio de Janeiro. <http://floradobrasil.jbrj.gov.br/jabot/floradobrasil/FB128483>. 05 Mar. 2015.

Prado J. 2015. Pteridaceae in Lista de Espécies da Flora do Brasil. Jardim Botânico do Rio de Janeiro. <http://floradobrasil.jbrj.gov.br/jabot/ floradobrasil/FB91802. 05 Mar. 2015.

Rizzini CT. 1992. Tratado de fitogeografia do Brasil. São Paulo, Hucitec.

Roderjan CV, GalvãoF, Kuniyoshi YS, Hatschbach GG. 2002. As unidades fitogeográficas do estado do Paraná. Ciência \& Ambiente 24: 75-92.
Rosseto EFS, Vieira AOS. 2013. Vascular Flora of the Mata dos Godoy State Park, Londrina, Paraná, Brazil. Check List 9: 1020-1034.

Rothfels JC, Sundue Ma, Kuo LY, et al. 2012. A revised family-level classifications for eupolypod II ferns (Polypodiidea: Polypodiales). Taxon 61: 515-533.

Sakagami CR. 2006. Pteridófitas do Parque Ecológico da Klabin, Telêmaco Borba, Paraná, Brasil. Msc Thesis, Universidade Federal do Paraná, Brazil.

Salino A, Almeida TE. 2009. Pteridófitas. In: Stehman JR, Forzza RC, Salino A, Sobral M, Costa DP, Kamino LHY. (eds.) Plantas da Floresta Atlântica. Rio de Janeiro, Jardim Botânico do Rio de Janeiro. p. 19-25.

Salino A, Mota NFO, Almeida TE. 2013. Lycophytes and monilophytes in Rio Preto State Park, Minas Gerais, Brazil. Acta Botanica Brasilica 27: $252-263$

Salino A, Silva SM, Dittrich VAO, Britezr M. 2005. Flora Pteridofítica. In: Marques MCM, Britez RM (eds.) História Natural e Conservação da Ilha do Mel. Curitiba, Editora UFPR. p. 85-101.

Salino A, Viveros RS. 2012. Flora II - As pteridófitas da Serra do Caraça. In: Christiano O. (ed.) Serra do Caraça. Belo Horizonte, V \& M do Brasil. p. 82-97.

Santiago ACP, Mynssen CM, Maureza D, Penedo TSA, Sfair JC. 2013. Dicksoniacae. In: Martinelli G, Moraes MA (eds.) Livro Vermelho da Flora do Brasil. Rio de Janeiro, Jardim Botânico do Rio de Janeiro. p. 475-476.

Santos ACC, Windisch PG. 2008. Análise da pteridoflora da área de proteção ambiental do morro da Borússia (Osório-RS). Pesquisas, Botânica 59: 237-252.

Schmitt JL, Fleck R, Burmeister EL, Rubio MAK. 2006. Diversidade e formas biológicas de pteridófitas da Floresta Nacional de Canela, Rio Grande do Sul: Contribuindo para o Plano de Manejo. Pesquisas, Botânica 57: 275-288.

Schwartsburd PB, Labiak PH. 2007. Pteridófitas do Parque Estadual de Vila Velha, Ponta Grossa, Paraná, Brasil. Hoehnea 34: 159-209.

Sehnem A. 1972. Pteridáceas. In: Reitz PR. (ed.) Flora Ilustrada Catarinense. Itajaí, Herbário Barbosa Rodrigues.

Senna RM, Kazmirczak C. 1997. Pteridófitas de um remanescente florestal no Morro da Extrema, Porto Alegre, RS. Revista da Faculdade de Zootecnia, Veterinária e Agronomia 4: 47-57.

Senna RM, Waechter JL. 1997. Pteridófitas de uma floresta com araucária. 1. Formas biológicas e padrões de distribuição geográfica. Iheringia Série Botânica 48: 41-58.

Silva JMC, Casteleti CHM. 2005. Estado da bodiversidade da Mata Atlântica brasileira. In: Galindo-Leal C, Câmara IG. (eds.) Mata Atlântica: Biodiversidade, ameaças e perspectivas. Belo Horizonte, Fundação SOS Mata Atlântica. p. 43-59

Smith AR, Pryer KM, Schuettpelz E, Korall P, Schneider H, Wolf PG. 2006. A classification for extant ferns. Taxon 55: 705-731.

SOS Mata Atlântica. 2015. A Mata Atlântica. http://www.sosma.org.br/ nossa-causa/a-mata-atlantica/.Brazil. 05 Mar. 2015.

Souza FSS, Salino A, Viana PL, Salimena FRG. 2012. Pteridófitas da Serra Negra, Minas Gerais, Brasil. Acta Botanica Brasilica 26: 378-390.

Stehman JR, Forzza RC, Salino A, Sobral M, Costa DP, Kamino LHY. 2009. Plantas da Floresta Mata Atlântica. Rio de Janeiro, Jardim Botânico do Rio de Janeiro.

Thiers B. 2015. Index Herbariorum: a global directory of public herbaria and associated staff. New York Botanical Garden's Virtual Herbarium. http://sweetgum.nybg.org/ih/. 05 Mar. 2015.

Tossulino MGP, Schaitza EG, Siqueira JDP, et al. 2007. Resumo Executivo da Avaliação Ecológica Rápida do Corredor Iguaçu-Paraná. http://www.redeprofauna.pr.gov.br/arquivos/File/biblioteca/livretoiguacu.pdf. 05 Mar. 2015.

Tryon RM, Tryon AF. 1982. Ferns and allied plants, with special reference to Tropical America. New York, Springer-Verlag.

Veloso HP, Rangel Filho ALR, Lima JCA. 1991. Classificação da vegetação brasileira adaptada a um sistema universal. Rio de Janeiro, IBGE Departamento de Recursos Naturais e Estudos Ambientais.

Zuquim G, Tuomisto H, Costa FRC, et al. 2012. Broad scale distribution of ferns and lycophytes along environmental gradients in Central and Northern Amazonia, Brazil. Biotropica 44: 752-762. 